Chirurg 2009 $\cdot 80: 497$

DOI 10.1007/s00104-008-1658-8

Online publiziert: 13. Mai 2009

๑) Springer Medizin Verlag 2009

J. Jähne ${ }^{1} \cdot$ J. Schirren ${ }^{2}$

${ }^{1}$ Zentrum Chirurgie Klinik für Allgemein- und Visceralchirurgie, Schwerpunkt für endokrine und onkologische Chirurgie, Diakoniekrankenhaus Henriettenstiftung gGmbH, Hannover

${ }^{2}$ Horst-Schmidt-Klinik, Wiesbaden

\title{
Drug-eluting Stents
}

\section{Ein unterschätztes Problem in der Chirurgie}

Die Zunahme der diagnostischen und therapeutischen Möglichkeiten hat nicht nur in der Chirurgie, sondern auch in der Kardiologie zu einer rasanten Weiterentwicklung geführt. Nach Etablierung der perkutanen transluminalen koronaren Angioplastie (PTCA) im Jahr 1979 haben die Koronarstents im weiteren Verlauf das Spektrum der interventionellen Therapiemöglichkeiten bedeutsam erweitert. Zur Vermeidung bzw. Reduzierung der nach PTCA und Stentimplantation auftretenden Restenosen wurden 2001 die „drug-eluting“ Stents (DES) in das kardiologische Interventionsarsenal eingeführt. Nicht zuletzt aufgrund eines erheblichen wirtschaftlichen Interesses der Medizinprodukteindustrie werden seitdem DES verstärkt - und vielleicht auch zu unkritisch (?) - implantiert.

\section{- Was bedeutet der verstärkte Einsatz von DES für die Chirurgie?}

Auf den ersten Blick erschließt sich dem in der Kardiologie als Laien zu bezeichnenden Chirurgen die innewohnende Problematik nicht. Bei genauerer Betrachtung allerdings wird die Herausforderung evident: Die DES-Implantation erfordert eine mindestens einjährige postinterventionelle Thrombozythenaggregationshemmung mit Aspirin und Clopidogrel. Das vorzeitige Absetzen geht mit einer sehr hohen Rate an tödlichen Myokardinfarkten einher. Der Chirurg befindet sich somit in einem therapeutischen Dilemma. Führt er eine Operation unter fortgesetzter Antikoagulation durch, riskiert er lebensbedrohliche chirurgische Blutungskomplikationen, wird die Antikoagulation abgesetzt, droht der Herztod!

Die vier nachfolgenden Artikel versuchen eine Annäherung an diese gegenwärtig noch unterschätzte Problematik und wollen eine Sensibilisierung der Chirurgen für diese Herausforderung anstreben. Der Fallbericht spiegelt sehr eindrucksvoll die z. T. dramatischen Verläufe wider und zeigt Möglichkeiten der Beherrschung solcher Situation auf, während der Beitrag von Bölükbas et al. die gegenwärtigen kardiologischen Empfehlungen und ihre Implikationen für die Chirurgie reflektiert. Darüber hinaus zeigt die herzchirurgische Arbeit in beeindruckender Weise die heutigen Möglichkeiten der modernen aortokoronaren Bypasschirurgie mit geringen Morbiditäts- und Mortalitätsraten als Alternative zur Stentimplantation auf, wobei natürlich auch die kardiologische Sichtweise zu berücksichtigen ist.

Wie auf vielen Gebieten der Chirurgie machen die DES die Notwendigkeit einer verstärkten interdisziplinären Zusammenarbeit deutlich. Waren früher wohl primär Kardiologen und Kardiochirurgen als Team gefordert, die bestmögliche Therapie für den einzelnen Patienten zu bestimmen, so müssen heute auch andere operative Fächer einbezogen werden, um das Risiko für den Patienten gerade im Langzeitverlauf bei der Notwendigkeit späterer operativer Eingriffe präziser zu definieren. Daneben sollten aber auch die juristischen Implikationen nicht verges- sen werden. Welcher Chirurg führt denn heute im Zeitalter gestiegener Haftungsprozesse elektive Operationen größeren Ausmaßes bei bestehender Antikoagulation durch? Die Zahl dürfte verschwindend gering sein. Neue Behandlungswege zwingen uns dazu, unsere bisherige Arbeitsweise zu überdenken und im interdisziplinären Dialog auf ein auch forensisch stabiles Fundament zu stellen.

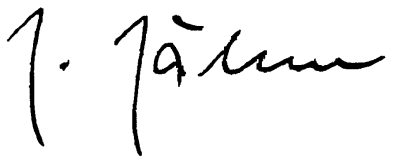

J. Jähne

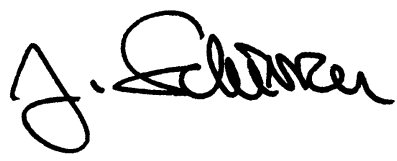

J. Schirren

\section{Korrespondenzadresse}

Prof. Dr. J. Jähne

Zentrum Chirurgie,

Klinik für Allgemein- und Visceralchirurgie,

Schwerpunkt für endokrine und onkologische

Chirurgie, Diakoniekrankenhaus

Henriettenstiftung $\mathrm{gGmbH}$,

Marienstraße 72-90, 30171 Hannover

joachim.jaehne@henriettenstiftung.de 